\title{
Passivity-Based Analysis and Performance Enhancement of a Vector Controlled VSC Connected to a Weak AC Grid
}

\author{
Adedotun J. Agbemuko, Member, IEEE, José Luis Domínguez-García, Member, IEEE, Oriol \\ Gomis-Bellmunt, Senior Member, IEEE, and Lennart Harnefors, Fellow, IEEE
}

\begin{abstract}
Despite attempts to increase the active power capability of vector-controlled voltage source converters (VSCs) connected to very weak grids, the interaction between the control dynamics and physical system is not completely understood. The result is often complex strategies that are difficult to implement. This paper proposes an intuitive modification of the VSC control based on physical considerations and dynamics of existing control. Several physical variables as seen from the point of common coupling (PCC) are found to contribute to the detrimental behavior of the VSC under weak connections. Hence, eliminating the impacts of these variables through feedforward eliminates their influence and significantly improves the active power capability. Notably, the basic structure of the vector controlled VSC is kept and its output-impedance is effectively reshaped. The proposed modification is validated through nonlinear time-domain simulations in MATLAB/Simulink Simscape Power System and results demonstrate the simplicity and intuitiveness of the modified structure.
\end{abstract}

Index Terms-VSC-HVDC, weak ac grid, passivity, impedance model.

\section{INTRODUCTION}

$\mathbf{T}$ HE voltage source converter (VSC) is currently the state-of-art of converter technology for high-voltage DC (HVDC) transmission [1]. One of the most important advantages of the VSC is its ability to connect to weak or unconventional grids, such as offshore ac grids [2], [3]. However, there are still certain challenges in connecting the VSC to weak grids (SCR $<3$ ), particularly VSCs based on the vector current control (VCC). According to literature, the stability limit of the vector-controlled VSC under very weak grids is severely limited to 0.6 per unit (pu) of converter rated power [4], [5].

One cause of such limits is the interaction between the phase-locked loop (PLL) of the VSC and dynamics at the

Adedotun J. Agbemuko and José Luis Domínguez-García are with the Electrical Power Systems Area, Institut de Rrecerca en Energia de Catalunya (IREC), 08930, Barcelona, Spain (e-mails: \{aagbemuko,jldominguez\}@irec.cat).

Oriol Gomis-Bellmunt is with the Electrical Engineering Department, Centre d'Innovaciò Tecnològica en Convertidors Estàtics i Accionaments (CITCEA-UPC), Universitat Politècnica de Catalunya, Barcelona 08028, Spain (e-mail:oriol.gomis@upc.edu).

Lennart Harnefors is with ABB Corporate Research Centre, 72178 Västerås, Sweden (e-mail:lennart.harnefors@se.abb.com).

This work was financially supported by the European Union's Horizon 2020 research and innovation programme under Marie-Sklodowska-Curie action INCITE - "Innovative controls for renewable source integration into smart energy systems", grant agreement No. 675318.

This work has also been partially funded by FEDER/Ministerio de Ciencia, Innovación y Universidades - Agencia Estatal de Investigación, Project RTI2018-095429-B-I00 and by the ICREA Academia program.
PCC under weak grid conditions, as reported in [6]-[8]. As a way to improve the performance of the PLL and overall system, different implementations and tuning methods have been proposed for the PLL [9]-[11]. In general, a reduced closed-loop bandwidth has been suggested to alleviate the impacts of PLL [12], [13]. Although this seems to provide relief under fairly low SCR, the active power capability is still limited. In a bid to eliminate the impacts of the PLL, the authors in [14]-[17] tackled the problem by eliminating the VCC and necessity for PLL. However, the PLL and inner current controller were suggested as backups.

Another potential cause of limited power capability is the complex interactions introduced by the outer loops of the vector controlled VSC [18]-[21]. The imbalance introduced by the direct voltage controller (DVC) was highlighted in [18] and means to mitigate control interactions were suggested. In [19] it was suggested to increase the closed-loop bandwidth of the alternating voltage controller (AVC) to improve the performance under weak ac grids. Notwithstanding, increasing the closed-loop bandwidth of the AVC without considering potential interactions with other loops still limits the capabilities. Seeing the complex couplings between the outer loops, EgeaAlvarez et al. in [20] proposed to decouple the loops on each axis of the VCC. This resulted in a quite complex implementation involving several controllers. In a simpler implementation, the authors in [22] proposed to simply add a feedforward loop using the active power to determine the reactive power requirements. Regardless, this computation depends on steadystate considerations that ignore the dynamics of control, and explicitly requires the grid impedance and its estimation.

In other efforts, it was proposed to improve the stability of the VSC under weak grids using an improved PCC voltage feedforward filter that explicitly considers the impacts of the PLL [23], [24]. Also, Davari et al. in [25] proposed a robust design of the conventional VCC by explicitly considering the impacts of the PLL in the design of the AVC. However, a direct consequence of including the impacts of the PLL in any design effort is that the order of an effective controller or filter may be as high as that of the entire VSC. Thus, implementation and scalability become complicated. To summarize, due to the lack of complete understanding of the dynamic interplays under weak grids, proposed solutions in literature are often complex and depend on the estimation of grid impedance.

As a concept to synthesize and control dynamical systems for robustness, passivity theory and more generally, dissi- 
pativity including in the frequency-domain is well applied [26]-[28]. More recently, passivity-based analysis and control design has been applied to grid-connected VSCs [18], [29][31]. The core of passivity theory as applied to interconnected systems relies on achieving an internal energy balance, and the interconnection of two passive subsystems is a passive system [32]. More generally, if one or more subsystems can be designed to dissipate energy (i.e. it is non-negative), there will be no destabilization [28]. Hence, for a grid-connected VSC, if the grid and VSC are both passive as subsystems, then the overall interconnected system is passive and thus stable [18], [33]. This is central to the research efforts on passivity for VSCs, where additional passivating components are added to the inner loop of $L$ connected VSCs [29], [30], and dual-loop $L C L$ connected VSCs [31]. However, not in the context of VSCs connected to very weak grids. Besides, in several cases, the goal is to passivate the VSC in the high-frequency region where the effects of a weak grid may not be felt. As opposed to previous works where consideration is given to the inner loop using passivating feedforward components, this article considers the outer loops using feedforward components that are better indicators of the detrimental behavior of the VSC under weak grids. Further, rather than to strictly passivate the VSC, this article attempts to eliminate major contributors to negativity. Thus, the dissipative property of the VSC is improved and enhancement in performance is obtained.

Notwithstanding the works done in [4], [9], [10], [12] considering the impacts of the PLL, we propose a method that keeps the basic structure of the VCC and conventional PLL, with applicability to any ac grid of arbitrary strength. In contrast to the suggestions proposed in [19] on increasing the bandwidth of the AVC, in this paper the reduction of the bandwidth of the DVC relative to that of the AVC is adopted. Additionally, we improve on the feedforward method described in [22] by considering the interaction between physical variables from the PCC and control dynamics without any requirement to explicitly estimate the grid impedance nor include it in the solution. Hence, the solution proposed is independent of grid impedance and without distance communication requirements unlike in [13] as measurements are local. The major contribution of this paper is an achievement of extended active power capabilities under weak grids by modifying the VSC, taking advantage of passivity analysis.

This paper is organized as follows. In Section II, an impedance modeling approach is adopted to derive the transfer functions of the VSC considering all control loops. Section III presents the concept of dissipativity and passivity analysis of the multi-input multi-output (MIMO) admittance to obtain insights into variables that contribute to detrimental behavior of the VSC. In Section IV, the results of passivity analysis is applied to modify the VSC outer-loops. Section V validates the analysis and modified capabilities through nonlinear timedomain simulations.

\section{II. $D Q$ Impedance TRANSFER Functions of the VSC}

The single-line diagram and control block layout of a typical vector controlled VSC station is shown in Fig. 1. The converter

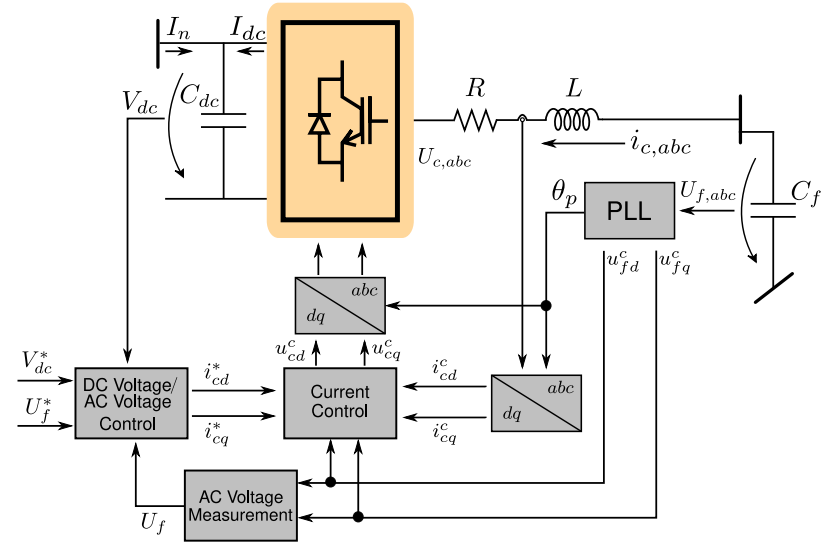

Fig. 1. VSC single-line terminal and control-block diagram

is modeled at the terminal level and assumed unconnected to any network to allow for the modularity of models. In the scope of this work, the filter bus of the converter is assumed to be the point of connection to the grid and the grid impedance is assumed to consist of passive $R, L$, and/or $C$ components.

\section{A. Current Controlled VSC}

The current controller (CC) of a VSC is responsible for the power exchange between the ac and dc sides, while ensuring current limits of components are not breached. The CC control structure is illustrated in Fig. 2. The open-loop circuit equations in grid reference that describe the dynamic behavior of the converter ac currents (physical system) in the synchronous reference frame can be expressed as

$$
L \frac{d i_{c d, q}}{d t}=u_{f d, q}-u_{c d, q}-R i_{c d, q}-j \omega_{1} L i_{c q, d}
$$

where $i_{c d, q}$ are the converter currents, $u_{c d, q}$ and $u_{f d, q}$ are the converter alternating and filter bus voltages respectively on $d$ and $q$ axes, $R$ and $L$ are the ac-side filter resistance and inductance respectively, and $\omega_{1}$ is the grid angular frequency. The ac output voltage is obtained from the $\mathrm{CC}$, in the converter reference frame as

$$
u_{c d, q}^{c}=-K_{c}(s)\left(i_{c d, q}^{*}-i_{c d, q}^{c}\right)-j \omega_{1} L i_{c q, d}^{c}+F_{d, q}(s) u_{f d, q}^{c}
$$

where $u_{c d, q}^{c}$ are the converter alternating voltages, $K_{c}(s)$ is the inner loop proportional-integral (PI) compensator, $i_{c d, q}^{c}$ are the converter ac currents, $u_{f d, q}^{c}$ are the filter voltages, and $F_{d, q}(s)$ are the PCC voltage feedforward filters. It is important to note that terms with superscript ' $c$ ' are converter $d q$ frame variables, and ' $*$ ' quantities are reference values. The PLL is responsible for the transformation between the grid (physical) and converter reference frames.

To include the impact of CC and PLL, the converter alternating voltage components can be eliminated from (1) by applying transformations from converter reference frame to grid reference through the PLL output angle. Subsequent to this, the linearized results are substituted into the linearized version of (1) in Laplace domain to obtain the impedance model with impact of PLL and CC included. Comprehensive derivation and details as applied in this work can be found in 


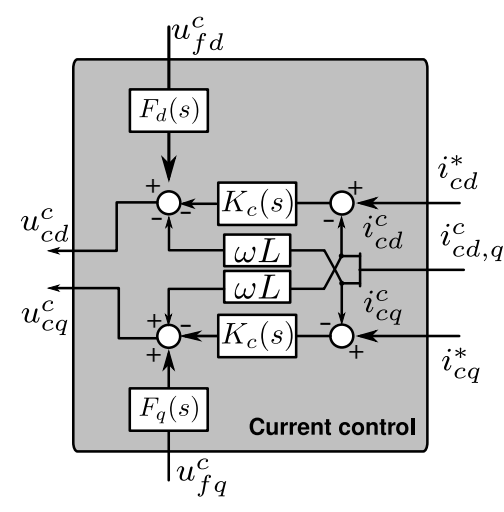

Fig. 2. Structure of inner current controller

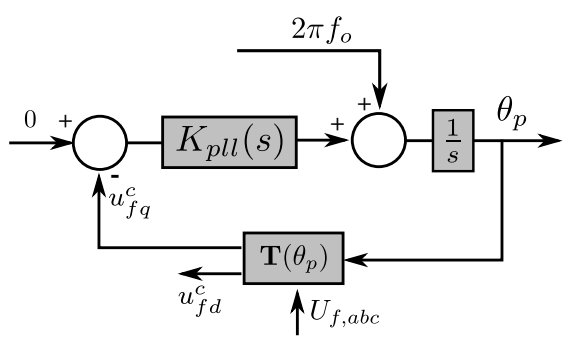

Fig. 3. PLL control-block diagram

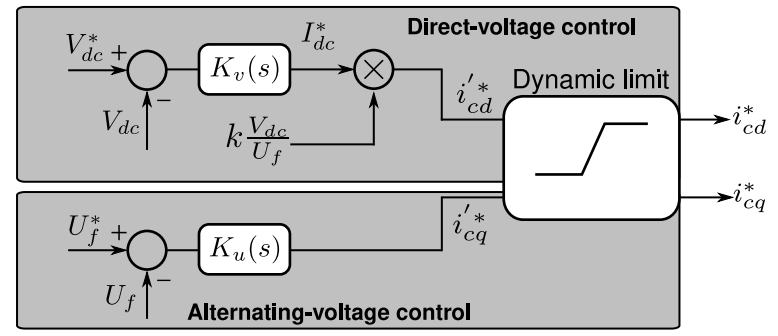

Fig. 4. Structure of the direct and alternating-voltage control

[34]. The impedance model of the VSC can be obtained as [18]

$$
\begin{aligned}
\left(\begin{array}{c}
\Delta i_{c d} \\
\Delta i_{c q}
\end{array}\right) & =\left(\begin{array}{cc}
h_{c l}^{i}(s) & 0 \\
0 & h_{c l}^{i}(s)
\end{array}\right)\left(\begin{array}{c}
\Delta i_{c d}^{*} \\
\Delta i_{c q}^{*}
\end{array}\right) \\
+ & \underbrace{\left(\begin{array}{cc}
y_{d d}(s) & y_{d q}(s) \\
y_{q d}(s) & y_{q q}(s)
\end{array}\right)}_{\mathbf{Y}_{a c}(s)}\left(\begin{array}{c}
\Delta u_{f d} \\
\Delta u_{f q}
\end{array}\right)
\end{aligned}
$$

where $h_{c l}^{i}(s)$ is the closed-loop reference to output transfer function, $\mathbf{Y}_{a c}(s)$ is the converter output admittance with the $\mathrm{CC}$ and PLL.

\section{B. Alternating-Voltage and Direct-Voltage Controlled VSC}

The control structure of the AVC and the DVC is illustrated in Fig. 4, showing the compensators and dynamic limiting function (neglected for linearized model). The AVC regulates the magnitude of the PCC-bus voltage to a constant value by providing the $q$-axis reference component to the CC. The DVC regulates the dc-bus voltage by providing the reference to the inner loop on the $d$-axis in a similar manner to the $q$-axis. The open-loop dynamic equation of the direct voltage is given as

$$
C_{d c} \frac{d V_{d c}}{d t}=I_{d c}+I_{n} ; \Longrightarrow V_{d c}=\frac{I_{d c}+I_{n}}{s C_{d c}}
$$

where $V_{d c}$ is the terminal direct voltage, $C_{d c}$ models the dc equivalent capacitance, $I_{d c}$ is the dc current injection from power balance, $I_{n}$ is the dc-side network current. The inner loop reference current on the $d$-axis can be obtained from the dc-side current injection reference as

$$
i_{c d}^{*}=k \frac{I_{d c}^{*} V_{d c}^{*}}{u_{f d}}
$$

where $k=2 / 3, V_{d c}^{*}$ is the reference direct voltage, $I_{d c}^{*}$ is the output of the DVC given as

$$
I_{d c}^{*}=K_{v}(s)\left(V_{d c}^{*}-V_{d c}\right)+I_{n}
$$

where $K_{v}(s)$ is the direct voltage PI compensator. Equation (6) can be linearized and substituted into the linearized version of (5) and results inserted into the $d$-axis equivalent of (3) to obtain

$$
\Delta i_{c d}=y_{d}(s) \Delta u_{f d}+y_{d q}(s) \Delta u_{f q}-y_{d c}(s) \Delta V_{d c}
$$

$y_{d}(s)=y_{d d}(s)-\frac{k h_{c l}^{i}(s) P^{0}}{\left(u_{f d}^{0}\right)^{2}}, \quad y_{d c}(s)=\frac{k K_{v}(s) h_{c l}^{i}(s) V_{d c}^{0}}{u_{f d}^{0}}$

where $h_{c l}^{i}(s)$ is the closed-loop reference to output transfer function of the inner loop, $y_{d}(s)$ and $y_{d c}(s)$ are auxiliary admittance functions, and $P^{0}$ is the active power operating point. In a similar manner as the direct voltage, $i_{c q}^{*}$ can be obtained directly from the AVC as

$$
i_{c q}^{*}=K_{u}(s)\left(U_{f}^{*}-U_{f}\right)
$$

where $U_{f}=\sqrt{\left(u_{f d}\right)^{2}+\left(u_{f q}\right)^{2}}$ is the filter bus voltage magnitude, $K_{u}(s)$ is the alternating voltage compensator. With the assumption that alternating voltage reference is constant, (8) is linearized around an operating point and substituted into the $q$-axis equivalent of (3) to obtain

$$
\begin{gathered}
\Delta i_{c q}=Y_{q d}(s) \Delta u_{f d}+Y_{q q}(s) \Delta u_{f q} \\
Y_{q d}(s)=\frac{y_{q d}(s) U_{f}^{0}-h_{c l}^{i}(s) K_{u}(s) u_{f d}^{0}}{U_{f}^{0}} \\
Y_{q q}(s)=\frac{y_{q q}(s) U_{f}^{0}-h_{c l}^{i}(s) K_{u}(s) u_{f q}^{0}}{U_{f}^{0}}
\end{gathered}
$$

where $Y_{q d}(s)$ and $Y_{q q}(s)$ are the measurable $q$-axis admittances of converter on the ac-side. To include the impacts of the dynamics of the direct voltage $\Delta V_{d c}$ in (7), the linearized power balance between ac and dc sides must be equated. Thus,

$$
\begin{array}{r}
\underbrace{\frac{1}{k}\left(u_{f d}^{0} \Delta i_{c d}+i_{c d}^{0} \Delta u_{f d}+i_{c q}^{0} \Delta u_{f q}+u_{f q}^{0} \Delta i_{c q}\right)}_{\Delta P_{a c}} \\
=\underbrace{V_{d c}^{0} \Delta I_{d c}+I_{d c}^{0} \Delta V_{d c}}_{\Delta P_{d c}}
\end{array}
$$


substituting $\Delta i_{c d}$ and $\Delta i_{c q}$ from (7) and (9) respectively, $\Delta I_{d c}$ can be isolated and substituted into the linearized open-loop dynamic equation of direct voltage in (4) to obtain

$$
\begin{gathered}
\Delta V_{d c}=H_{d d}(s) \Delta u_{f d}+H_{q q}(s) \Delta u_{f q}+Z_{o c}(s) \Delta I_{n} \\
H_{d d}(s)=\frac{u_{f d}^{0} y_{d}(s)+u_{f q}^{0} Y_{q d}(s)+i_{c d}^{0}}{k\left(s C_{d c} V_{d c}^{0}+I_{d c}^{0}\right)+u_{f d}^{0} y_{d c}(s)} \\
H_{q q}(s)=\frac{u_{f d}^{0} y_{d q}(s)+u_{f q}^{0} Y_{q q}(s)+i_{c q}^{0}}{k\left(s C_{d c} V_{d c}^{0}+I_{d c}^{0}\right)+u_{f}^{d 0} y_{d c}(s)} \\
Z_{o c}(s)=\frac{k V_{d c}^{0}}{k\left(s C_{d c} V_{d c}^{0}+I_{d c}^{0}\right)+u_{f d}^{0} y_{d c}(s)}
\end{gathered}
$$

where $H_{d d}(s)$ and $H_{q q}(s)$ are the interaction transfer functions between the filter voltage components on either axes and the direct voltage, and $Z_{o c}(s)$ is the imposed dc-side impedance. Back-substituting $\Delta V_{d c}$ into (7), the reshaped ac-side admittance considering the direct voltage dynamics and control is obtained as

$$
\begin{gathered}
\Delta i_{c d}=Y_{d d}(s) \Delta u_{f d}+Y_{d q}(s) \Delta u_{f q}+H_{d c}(s) \Delta I_{n} \\
Y_{d d}(s)=y_{d}(s)-H_{d d}(s) y_{d c}(s) \\
Y_{d q}(s)=y_{d q}(s)-H_{q q}(s) y_{d c}(s) \\
H_{d c}(s)=Z_{o c}(s) y_{d c}(s)
\end{gathered}
$$

where $H_{d c}(s)$ is the interaction transfer function between the converter ac and dc currents. With respect to measurable admittances, equations (9) and (12) describe the complete input-output behavior of the alternating and direct voltage controlled VSC at the terminal level on the ac-side. This can be represented in a matrix form as

$$
\mathbf{Y}(s)=\left(\begin{array}{cc}
Y_{d d}(s) & Y_{d q}(s) \\
Y_{q d}(s) & Y_{q q}(s)
\end{array}\right)
$$

where $\mathbf{Y}(s)$ is a complex asymmetrical MIMO admittance of the converter. The detailed modeling procedures as adopted in this paper can be found in [34].

\section{Model Verification}

To validate the accuracy of the analytical derivations carried out in the previous section, a detail nonlinear model is developed in MATLAB/Simulink Simscape Power System toolbox with system and control data presented in Tables I and II. For a VSC in inverter mode with an active power operating point of $-200 \mathrm{MW}(0.25 \mathrm{pu})$, Fig. 5 shows a comparison of the analytical admittance frequency response and measurements from simulation. It can be observed in general that there is a good match between the analytical derivation (solid blue) and detailed simulation (dotted red) within expected accuracy.

\section{Dissipativity/Passivity-Based Analysis of VSC ADMitTANCE}

\section{A. Dissipativity of Dynamic Systems}

Passivity and dissipativity as a generalization of passivity are both fundamental properties of dynamic systems related to energy [35]. Both properties characterize the implicit energy
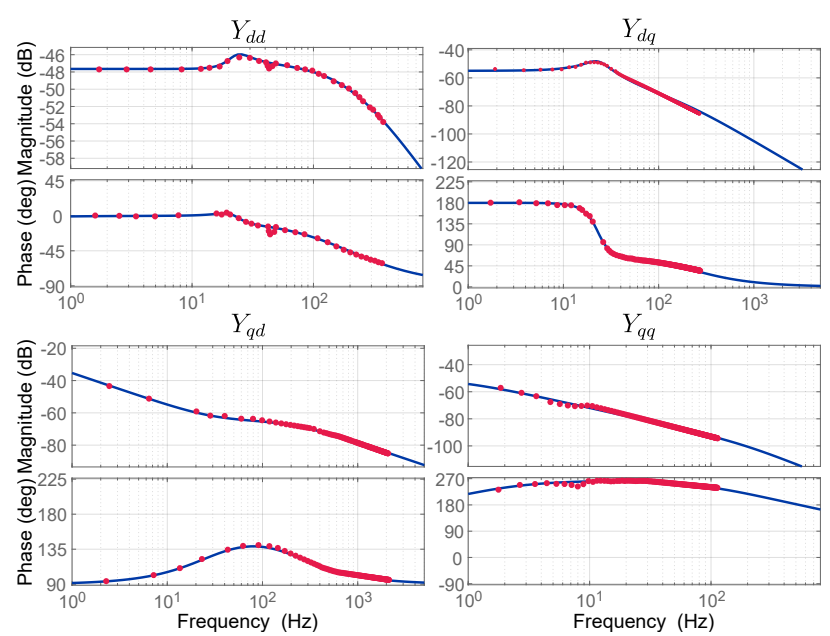

Fig. 5. VSC output admittance validation. Analytical (solid blue), simulation measurement (dotted red)

TABLE I

GRID PARAMETERS

\begin{tabular}{cc}
\hline Parameter & Value \\
\hline Rated power & $800 \mathrm{MVA}$ \\
Rated AC-side voltage & $400 \mathrm{kV}$ \\
Rated AC-side voltage & $220 \mathrm{kV}(\mathrm{rms} \mathrm{L}-\mathrm{L})$ \\
AC Filter impedance & $0.004+j 0.1 \mathrm{pu}$ \\
Transformer impedance & $j 0.12 \mathrm{pu}$ \\
Equivalent DC capacitance & $150 \mu \mathrm{F}$ \\
Capacitive reactance & $8.75 \mathrm{pu}$ \\
$X_{g} / R_{g}$ ratio & 10 \\
\hline
\end{tabular}

TABLE II

Control Parameters

\begin{tabular}{ccc}
\hline Controller & Time constant & Damping ratio \\
\hline AC-side Current & $1.5 \mathrm{~ms}$ & 0.7071 \\
DC-side voltage & $22.5 \mathrm{~ms}$ & 0.7071 \\
AC-side voltage & $100 \mathrm{~ms}$ & 0.7071 \\
Phase-locked loop (PLL) & $100 \mathrm{~ms}$ & 0.7071 \\
\hline
\end{tabular}

consumption and are closely related to the stability of interconnected systems [27]. Considering a linear time-invariant (LTI) continuous system, its dissipative properties can be determined by constructing a quadratic function (differential and otherwise) with respect to its input/output variables [28]. If this function is positive, then the system dissipates energy and destabilization will not occur [33]. For a VSC interconnected to a passive AC grid composed of $R L C$ components, the passive/dissipative properties of the entire interconnected system will depend on properties of the VSC.

Considering a VSC, the quadratic function is an input/output transfer function that can be constructed in terms of power dissipation according to [18]

$$
P=v^{H} i+i^{H} v
$$

where $H$ is the Hermitian operator, $v$ and $i$ are complex phasors for voltages and currents respectively [36]. As a 
prescribed function of frequency, $i=\mathbf{Y}(j \omega) v$. Then,

$$
\begin{aligned}
v^{H} i+i^{H} v & =v^{H} \mathbf{Y}(j \omega) v+[\mathbf{Y}(j \omega) v]^{H} v \\
& =v^{H}\left[\mathbf{Y}(j \omega)+\mathbf{Y}^{H}(j \omega)\right] v
\end{aligned}
$$

with

$$
\mathbf{Y}(j \omega)+\mathbf{Y}^{H}(j \omega)=\left[\begin{array}{cc}
A & C^{*} \\
C & B
\end{array}\right]
$$

with elements

$$
\begin{aligned}
A & =2 \operatorname{Re}\left\{Y_{d d}(j \omega)\right\}, \quad B=2 \operatorname{Re}\left\{Y_{q q}(j \omega)\right\} \\
C C^{*} & =\left(Y_{d q}^{*}(j \omega)+Y_{q d}(j \omega)\right)\left(Y_{d q}(j \omega)+Y_{q d}^{*}(j \omega)\right) \\
& =\operatorname{Re}\left\{Y_{d q}(j \omega)+Y_{q d}(j \omega)\right\}^{2}+\operatorname{Im}\left\{Y_{d q}(j \omega)-Y_{q d}(j \omega)\right\}^{2} .
\end{aligned}
$$

The above is the required quadratic function [18], [28] and for a linearized VSC, the differential quadratic form applies. The criterion establishes that if $\mathbf{Y}(j \omega)+\mathbf{Y}^{H}(j \omega)>0$ (i.e. passive), the system dissipates energy. That is, if the VSC can be designed to dissipate power at all frequencies, there will be no destabilization. However, it is impossible to guarantee passivity across all frequencies for a VSC. Therefore, the goal here is not to make the VSC strictly passive; rather, to improve its dissipative properties by eliminating variables that significantly contribute to the negativity of $\mathbf{Y}(j \omega)+\mathbf{Y}^{H}(j \omega)$. In other words, simply reducing the area of negative conductance is sufficient to significantly enhance the performance of a vector controlled VSC.

The check of dissipative properties of the matrix to the right of (16) is simply a check of positivity described by [37]

$$
A>0, \quad B>0, \quad A B>C^{*} C,
$$

and due to the squares involved, $C^{*} C \geq 0$. Following the expressions in (17) and the above conditions, it can be established that it is desired to have $C C^{*} \approx 0$. Any $C^{*} C \gg 0$ puts a higher minimum bound on $A B$; hence, this imposes difficulty on efforts to reduce the negativity or improve the positivity of the system.

\section{B. Decomposition of $\mathbf{Y}(s)$}

The $d d, q q, d q$, and $q d$ elements of the VSC admittance $\mathbf{Y}(s)$ are further decomposed into individual components for ease of insights. Due to the order of the system as modeled, several assumptions are made in the following to keep the problem compact and to work as symbolically as possible. Where necessary, the impacts of assumptions are indicated.

- The $q$-axis PCC voltage at an operating point $u_{f q}^{0} \approx 0$. This holds for nearly all cases and in the worst case, its impact almost always appear as $u_{f q}^{0} / U_{f}^{0}$ which is negligible. Hence, $P^{0}=u_{f d}^{0} i_{c d}^{0} / k$.

- The PLL deviation angle at an operating point, $\theta_{p}^{0}=0$. This follows from previous assumption.

- For the closed-loop reference to output transfer function of the inner loop given as

$$
h_{c l}^{i}(s)=\frac{K_{c}(s) y_{a c}(s) \cos \theta_{p}^{0}}{1+K_{c}(s) y_{a c}(s)},
$$

following from previous assumption $\cos \theta_{p}^{0}=1$ and for a good design, $K_{c}(s) y_{a c}(s) \gg 1$ (up to the cut-off frequency). Therefore, the magnitude of the closed-loop transfer function $h_{c l}^{i}(s)=1$. This holds up till the cut-off frequency chosen to be around $106 \mathrm{~Hz}(\approx 1.5 \mathrm{~ms}$ time response).

1) $Y_{d d}(s)$ : Applying the assumptions above and substituting the expressions for $y_{d}(s), y_{d c}(s), H_{d d}(s)$ from (7) into (12), $Y_{d d}(s)$ can be decomposed as

$$
Y_{d d}(s)=a_{d d 1}(s)\left(F_{d}(s)-1\right)+a_{d d 2}(s)\left(1-F_{d}(s)\right)-a_{d d 3}
$$

with coefficients

$$
\begin{aligned}
& a_{d d 1}(s)=\frac{y_{a c}(s) K_{v}(s) V_{d c}^{0}}{\left(K_{c}(s) y_{a c}(s)+1\right)\left(s C_{d c} V_{d c}^{0}+K_{v}(s) V_{d c}^{0}+I_{d c}^{0}\right)} \\
& a_{d d 2}(s)=\frac{y_{a c}(s)}{K_{c}(s) y_{a c}(s)+1}, \quad a_{d d 3}=\frac{i_{c d}^{0}}{u_{f d}^{0}}
\end{aligned}
$$

where $y_{a c}(s)$ is the primitive admittance of the VSC consisting of $R$ and $L$. To guarantee

$$
\operatorname{Re}\left\{Y_{d d}(j \omega)\right\} \geq 0 \quad \forall \omega
$$

then, from (20)

$$
\begin{aligned}
\Longrightarrow\left[\operatorname{Re}\left\{a_{d d 1}(j \omega)\right\} \cdot \operatorname{Re}\left\{F_{d}(j \omega)-1\right\}\right. \\
\\
\left.-\operatorname{Im}\left\{a_{d d 1}(j \omega)\right\} \cdot \operatorname{Im}\left\{F_{d}(j \omega)\right\}\right] \\
+\left[\operatorname{Re}\left\{a_{d d 2}(j \omega)\right\} \cdot \operatorname{Re}\left\{1-F_{d}(j \omega)\right\}\right. \\
\left.-\operatorname{Im}\left\{a_{d d 2}(j \omega)\right\} \cdot \operatorname{Im}\left\{F_{d}(j \omega)\right\}\right]-a_{d d 3} \geq 0 .
\end{aligned}
$$

For an all-pass feedforward filter

$$
\operatorname{Re}\left\{F_{d}(j \omega)\right\}=1 ; \quad \operatorname{Im}\left\{F_{d}(j \omega)\right\}=0, \quad \forall \omega
$$

therefore, $a_{d d 1}$ and $a_{d d 2}$ cancel out in (22). Clearly, the real part of $Y_{d d}(j \omega)$ would depend on $-a_{d d 3}$. Hence, in rectification (where $i_{c d}^{0}>0$ ), $\operatorname{Re}\left\{Y_{d d}(j \omega)\right\}<0$, whereas in inversion (where $i_{c d}^{0}<0$ ), $\operatorname{Re}\left\{Y_{d d}(j \omega)\right\}>0$. For a first order low pass filter with bandwidth less than or equal to that of the CC, Fig. 6a shows the impacts of varying cut-off frequencies of the feedforward filter $F_{d}(s)$ on the $d$-axis component at rated operating point for rectifier operation $\left(P^{0}=1 \mathrm{pu}\right)$. It can be seen that the conductance $\operatorname{Re}\left\{Y_{d d}(j \omega)\right\}<0$ for all frequencies for an all-pass feedforward filter. Whereas, for a feedforward filter with a cut-off frequency less than the CC bandwidth, $\operatorname{Re}\left\{Y_{d d}(j \omega)\right\}>0$ for frequencies beyond the synchronous region. However, below the synchronous frequency, the feedforward filter introduces more negativity compared to an all-pass filter in the same region. This is the same region where the effect of a weak grid is most severe. Likewise for inverter operation at rated operating point $\left(P^{0}=-1 \mathrm{pu}\right)$ shown in Fig. 6b, an all-pass filter guarantees $\operatorname{Re}\left\{Y_{d d}(j \omega)\right\}>0$ for all frequencies. However, for any feedforward filter with a cut-off frequency less than the bandwidth of the $\mathrm{CC}$, the filter introduces negativity into an otherwise positive component.

In summary, the feedforward filter introduces detrimental interactions below the synchronous frequency, despite significant improvements they provide above it. A simple solution is to adopt an all-pass filter under weak grid conditions in addition to other potential solutions. 


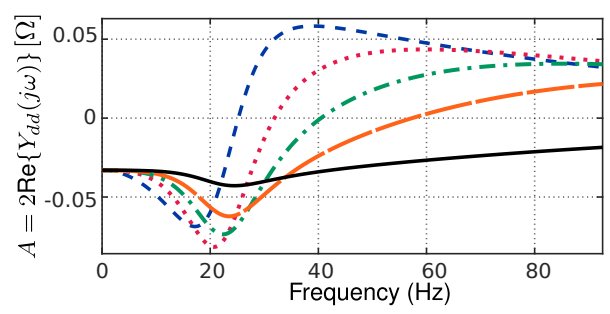

(a)

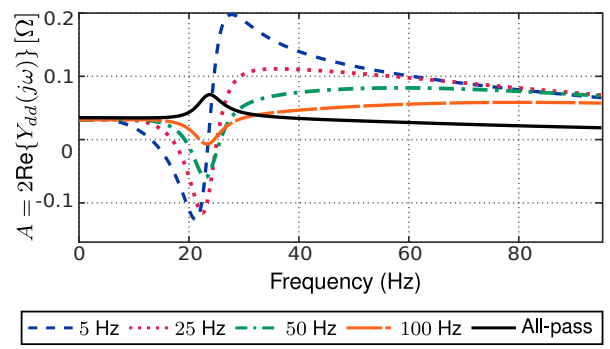

(b)

Fig. 6. Impact of varying cut-off frequency of feedforward filter on $A$ (a) rectification (b) inversion

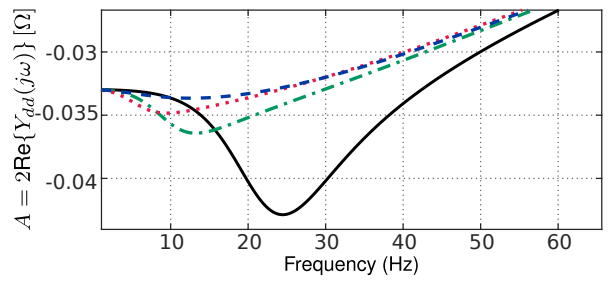

(a)

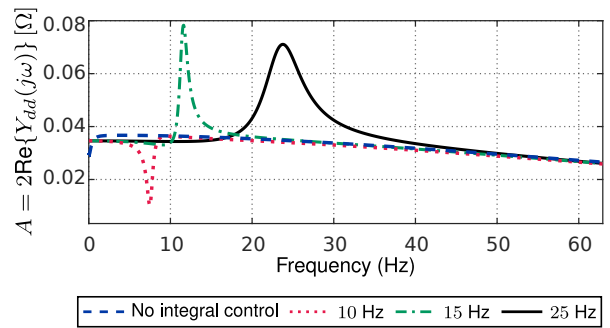

(b)

Fig. 7. Impact of closed-loop bandwidth of the DVC on $A=2 \operatorname{Re}\left\{Y_{d d}(j \omega)\right\}$ (a) rectification (b) inversion

For a case where $u_{f q}^{0} \neq 0$ with an all-pass feedforward filter, $Y_{d d}(s)$ can be decomposed as

$$
Y_{d d}(s)=\frac{K_{u}(s) K_{v}(s) V_{d c}^{0} u_{f q}^{0}}{\left(s C_{d c} V_{d c}^{0}+K_{v}(s) V_{d c}^{0}+I_{d c}^{0}\right) U_{f}^{0}}-a_{d d 3}
$$

without further simplifications, it can be easily conjectured from (24) that reducing the bandwidth of the DVC and increasing the bandwidth of the AVC may reduce the area of negative conductance. However, this depends on the actual value of $u_{f q}^{0}$, the lower the value, the less influence the AVC has on passivity of the system.

The impacts of varying the closed-loop bandwidths of the DVC with an all-pass filter while the bandwidth of the AVC is kept constant in rectification is shown in Fig. 7a. It can be seen that, although the conductance is negative for all frequencies, in the near synchronous region, the faster the bandwidth of the

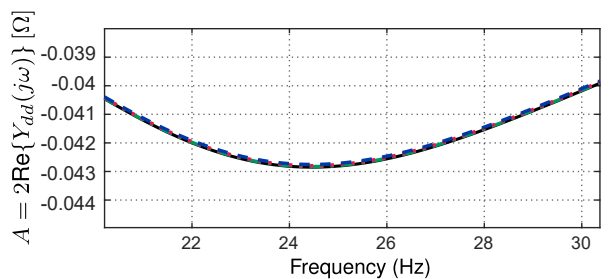

(a)

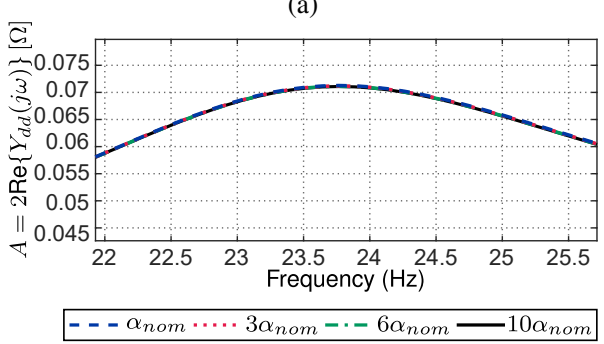

(b)

Fig. 8. Impact of varying closed-loop bandwidth $\alpha_{u}$ of the AVC on $A=$ $2 \operatorname{Re}\left\{Y_{d d}(j \omega)\right\}$ (a) rectification (b) inversion

DVC, the more negative the conductance becomes. Moreover, if an acceptable operation can be guaranteed without the integral gain, the resulting reduction in negative area can improve the behavior. In the inversion stage as shown in Fig. $7 \mathrm{~b}$, the conductance remains positive as long as an all-pass filter is employed. A suggestion is to adopt a slower DVC bandwidth at a cost of performance degradation of the direct voltage, however, a compromise could be found.

The impacts of varying the closed AVC bandwidth in rectification and inversion while keeping the bandwidth of the DVC constant is shown in Fig. 8. In both power stages, the impact of the AVC bandwidth is quite negligible (even for $u_{f q}^{0} \neq 0$ ) at least on the $d$-axis .

2) $Y_{q q}(s)$ : Applying the assumptions as previously, and evaluating $Y_{q q}(s)$ as given in (9)

$$
\begin{aligned}
Y_{q q}(s) & =y_{q q}(s) \\
& =a_{q q 1}(s)+a_{q q 2}(s)\left(1-F_{q}(s)\right)+a_{q q 3}(s)\left(u_{f d}^{0}-u_{c d}^{0}\right)
\end{aligned}
$$

with coefficients

$$
\begin{aligned}
& a_{q q 1}(s)=\frac{y_{a c}(s) K_{c}(s) K_{p l l}(s) i_{c d}^{0}}{\left(K_{c}(s) y_{a c}(s)+1\right)\left(u_{f d}^{0} K_{p l l}(s)+s\right)} \\
& a_{q q 2}(s)=\frac{y_{a c}(s) s}{\left(K_{c}(s) y_{a c}(s)+1\right)\left(u_{f d}^{0} K_{p l l}(s)+s\right)} \\
& a_{q q 3}(s)=\frac{y_{a c}(s) K_{p l l}(s)}{\left(K_{c}(s) y_{a c}(s)+1\right)\left(u_{f d}^{0} K_{p l l}(s)+s\right)}
\end{aligned}
$$

where $u_{c d}^{0}$ is the converter alternating voltage on the $d$-axis. To guarantee $\operatorname{Re}\left\{Y_{q q}(j \omega)\right\} \geq 0$

$$
\begin{gathered}
\Longrightarrow \operatorname{Re}\left\{a_{q q 1}(j \omega)\right\}+\left[\operatorname{Re}\left\{a_{q q 2}(j \omega)\right\} \cdot \operatorname{Re}\left\{1-F_{q}(j \omega)\right\}\right. \\
\left.-\operatorname{Im}\left\{a_{q q 2}(j \omega)\right\} \cdot \operatorname{Im}\left\{F_{q}(j \omega)\right\}\right] \\
+\operatorname{Re}\left\{a_{q q 3}(j \omega)\right\}\left(u_{f d}-u_{c d}\right) \geq 0 .
\end{gathered}
$$

For an all-pass filter, $a_{q q 2}$ cancels out. For a case where $u_{f}^{q 0} \neq$ 0 , an additional term $\left(\frac{K_{u}(s) u_{f q}^{0}}{U_{f}^{0}}\right)$ adds to $Y_{q q}(s)$, however, 


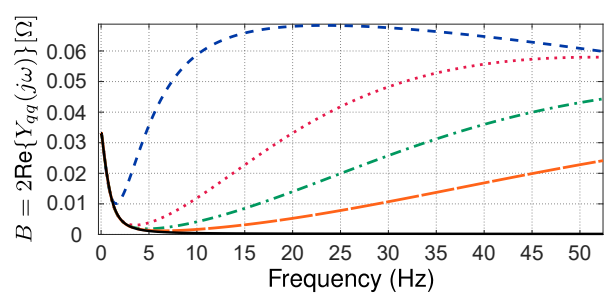

(a)

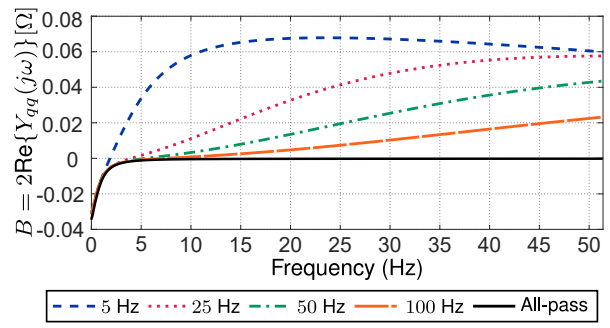

(b)

Fig. 9. Detailed view below $2 f_{1}$ for impact of varying cut-off frequency of feedforward filter on $B=2 \operatorname{Re}\left\{Y_{q q}(j \omega)\right\}$ when $\left(u_{f d}^{0}-u_{c d}^{0}>0\right)$ (a) rectification (b) inversion

small enough to be negligible. Therefore,

$$
\begin{aligned}
& \operatorname{Re}\left\{Y_{q q}(j \omega)\right\} \geq 0 \Longleftrightarrow \\
& \operatorname{Re}\left\{a_{q q 1}(j \omega)\right\} \geq 0, \quad\left(u_{f d}^{0}-u_{c d}^{0}\right) \geq 0 .
\end{aligned}
$$

$\operatorname{Re}\left\{a_{q q 1}(j \omega)\right\}$ is dependent on active power operating point, whereas the difference $\left(u_{f d}-u_{c d}\right)$ is dependent on the reactive power requirements demanded of the converter under any grid conditions. This sets the major difference between passivity of $Y_{d d}(s)$ and $Y_{q q}(s)$ components, where the former depends mainly on active power operating point, but the latter on both active power and reactive power demands in any direction.

More importantly, under fairly strong grids where the reactive power requirements can be easily managed and fairly constant across operating points, the difference $\left(u_{f d}-u_{c d}\right)$ is positive. In rectification, if the difference is negative at all, under such conditions the negativity is mitigated by the positivity of $a_{q q 1}(s)$; thus, $\operatorname{Re}\left\{Y_{q q}(j \omega)\right\}>0$. However, under weak grids, the difference becomes negative with increasing active power due to severe reactive power requirements demanded of the converter. Hence, the negativity increases with increasing active power leading to instability when the positivity due to $a_{q q 1}(s)$ (since its positivity is limited by rated current) is lower than the negativity due to $\left(u_{f d}-u_{c d}\right)$. In inversion, the negative sign of $i_{c d}^{0}$ makes $\operatorname{Re}\left\{Y_{q q}(j \omega)\right\}<0$, and this is further compounded by the negativity of $\left(u_{f d}-u_{c d}\right)$ under weak grids.

The impacts of varying cut-off frequencies on the feedforward filter on the $q$-axis component in the rectification stage when the difference $\left(u_{f d}^{0}-u_{c d}^{0}\right)>0$ is shown in Fig. 9a. It is shown that $\operatorname{Re}\left\{Y_{q q}(j \omega)\right\}>0$ for all frequencies of interest and for any feedforward filter due to $\operatorname{Re}\left\{a_{q q 1}(j \omega)\right\}>0$ and $\operatorname{Re}\left\{a_{q q 3}(j \omega)\right\}>0$. Whereas in inversion as shown in Fig. $9 \mathrm{~b}, \operatorname{Re}\left\{Y_{q q}(j \omega)\right\}<0$ for all frequencies with an all-pass filter, however, significantly less negative with a low cut-off frequency feedforward filter. This generally suggests that a feedforward filter should be adopted. However, due to potential negativity of $\left(u_{f d}-u_{c d}\right)$ under very weak grid conditions and high power, the feedforward filter only marginally improves the capabilities.

3) $Y_{d q}(s)$ and $Y_{q d}(s)$ : According to (16) both $Y_{d q}(s)$ and $Y_{q d}(s)$ contribute to $C C^{*}$. From equation (12), $Y_{d q}(s)$ can be decomposed as

$$
Y_{d q}(s)=-a_{d q 1}(s) \frac{V_{d c}^{0} i_{c q}^{0}}{u_{f d}^{0}}+a_{d q 2}(s) u_{c q}^{0}-a_{d q 3}(s) i_{c q}^{0}
$$

with coefficients

$$
\begin{gathered}
a_{d q 1}(s)=\frac{K_{v}(s)\left(s\left(K_{c}(s) y_{a c}(s)+1\right)+K_{p l l}(s) u_{f d}^{0}\right)}{\left(K_{p l l}(s) u_{f d}^{0}+s\right)\left(K_{c}(s) y_{a c}(s)+1\right)} \\
a_{d q 2}=\frac{\left(V_{d c}^{0}\left(s C_{d c}+K_{v}(s)\right)+I_{d c}^{0}\right)}{\left(K_{p l l}(s) u_{f d}^{0}+s\right)\left(K_{c}(s) y_{a c}(s)+1\right)} \\
a_{d q 3}(s)=\frac{\left(V_{d c}^{0}\left(s C_{d c}+K_{v}(s)\right)+I_{d c}^{0}\right)}{\left(K_{p l l} u_{f d}^{0}+s\right)\left(K_{c}(s) y_{a c}(s)+1\right)}
\end{gathered}
$$

It is clear that $Y_{d q}(s)$ depends on a complex interplay between reactive power, DVC, and PLL closed-loop control. Similarly, applying the assumptions and evaluating $Y_{q d}(s)$ as given in (9)

$$
Y_{q d}(s)=-K_{u}(s)
$$

clearly, $Y_{q d}(s)$ depends on the closed AVC compensator $K_{u}(s)$. Particularly, $\operatorname{Re}\left\{Y_{q d}(j \omega)\right\}$ depends on the proportional gain of the alternating voltage compensator, and $\operatorname{Im}\left\{Y_{q d}(j \omega)\right\}$ depends on the integral gain.

It was previously mentioned that a lower bound on $C C^{*}$ directly results in a lower bound on $A B$ and hence, the relative ease of improving the overall passivity. To obtain $C C^{*} \approx 0$, it is required that

$$
\begin{aligned}
& \left(\operatorname{Re}\left\{Y_{d q}(j \omega)\right\}+\operatorname{Re}\left\{Y_{q d}(j \omega)\right\}\right)^{2} \approx 0 \\
& \left(\operatorname{Im}\left\{Y_{d q}(j \omega)\right\}-\operatorname{Im}\left\{Y_{q d}(j \omega)\right\}\right)^{2} \approx 0 .
\end{aligned}
$$

It was suggested from the passivity checks in the previous case to adopt a low bandwidth DVC to reduce the negativity of the system. For a reduced DVC bandwidth to around $15 \mathrm{~Hz}$, Fig. 10 depicts the impacts of varying PLL closed-loop bandwidths on the off-diagonal components in rectification and inversion respectively. In rectification as shown in Fig. 10a, it can be seen that $C C^{*}$ is very low $(\approx 0)$. In inversion as shown in Fig. $10 \mathrm{~b}$, increasing peaks can be seen around the bandwidth of the DVC as the PLL closed-loop bandwidth is increased. That is, a reduced DVC bandwidth puts stringent conditions on the diagonal elements if a faster PLL bandwidth is required. This is contrary to the benefits of the reduction of the bandwidth for the DVC from the case of the $Y_{d d}$. This is the result of a resonance between the DVC and the PLL as acknowledged in [38]. As such, a change in closed-loop bandwidth of the DVC moves the resonance; whereas a change in closed-loop bandwidth of the PLL increases or decreases the severity of the resonance. Hence, a slow bandwidth PLL should be adopted to obtain $C C^{*} \approx 0$ as much as possible. Otherwise, the dynamics 


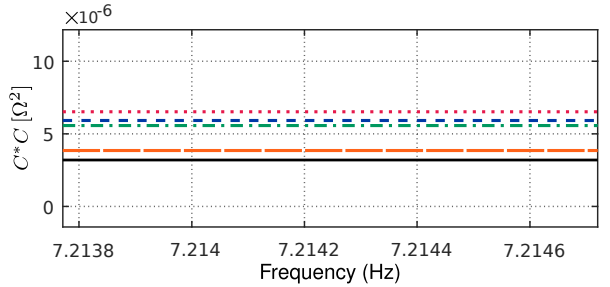

(a)

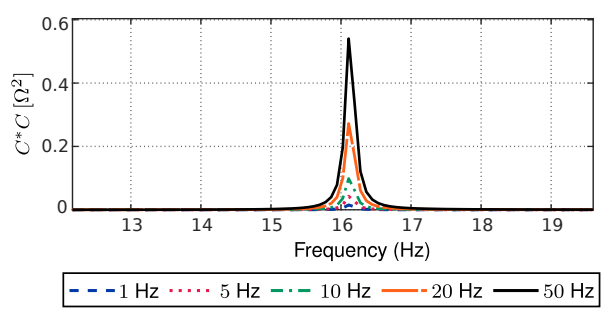

(b)

Fig. 10. Detailed view below $f_{1}$ for varying PLL closed-loop bandwidths on $C C^{*}$ (a) rectification (b) inversion

of the direct voltage could be improved with additional terms such as a damping loop if a faster PLL is desired.

\section{Proposed Modification of Vector Control}

Physically, the main control challenge of a VSC under a weak grid is an effort by the converter to force the AVC to perform two strongly coupled functions-PCC voltage magnitude control and reactive power management through the same control channel and input. In the same vein, the bandwidth of the DVC may constrain the performance of the AVC, especially under weak grids.

The major proposition involves an additional open-loop feedforward (on the $q$-axis of outer loop) of the difference $\left(u_{f d}-u_{c d}\right)$ thereby decoupling the discussed functions. This difference implicitly encodes the actual grid impedance and reactive current required relative to the operating point, without the requirement to estimate the grid impedance as suggested in literature. In addition, due to the effects of reducing the bandwidth of the DVC as analysed, a bandpass filter may be adopted to mitigate ac and dc sides interactions. The described modification of outer loops of vector current control is shown in Fig. 11. Under strong grids, this acts as a redundant loop to the AVC as both share the reactive current requirements. The overall modification on both axis can be described by

$$
\begin{aligned}
& i_{c d}^{*}=\left(V_{d c}^{*}-V_{d c}\right)\left(K_{v}(s)+F_{v}(s) k_{f v}\right) \\
& i_{c q}^{*}=K_{u}(s)\left(U_{f}^{*}-U_{f}\right)+F_{u}(s)\left(u_{f d}-u_{c d}\right) k_{f u}
\end{aligned}
$$

where $k_{f v}$ and $k_{f u}$ are constant gains, $F_{v}(s)$ is a bandpass filter, and $F_{u}(s)$ is a low pass filter with structures described by

$$
F_{v}(s)=\frac{\frac{\omega_{v 0}}{Q} s}{s^{2}+\frac{\omega_{v 0}}{Q} s+\omega_{v 0}^{2}}, \quad F_{u}(s)=\frac{\omega_{u}}{s+\omega_{u}}
$$

where $\omega_{v 0}$ is the midpoint of frequencies of interest, and $\omega_{u}$ is the cut-off frequency of the low pass filter $F_{u}(s)$ of the additional feedforward term $\left(u_{f d}-u_{c d}\right) ; \omega_{u}$ should be chosen well below the bandwidth of the inner loop.

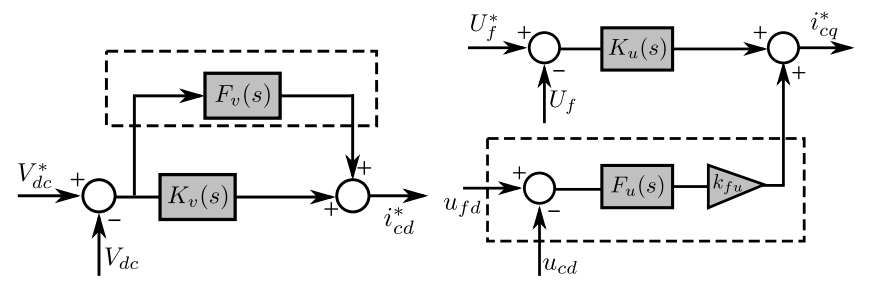

(a)

(b)

Fig. 11. Modified outer loops of vector current control (a) $d$-axis modification (b) $q$-axis modification
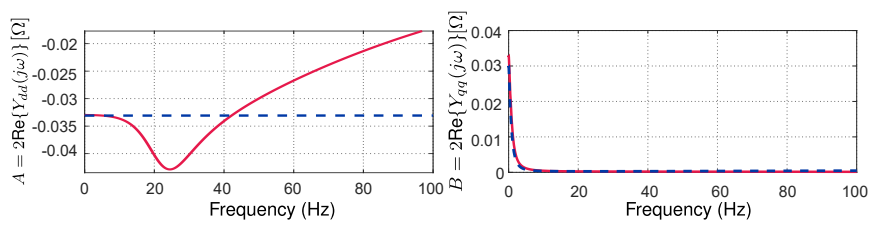

(a)
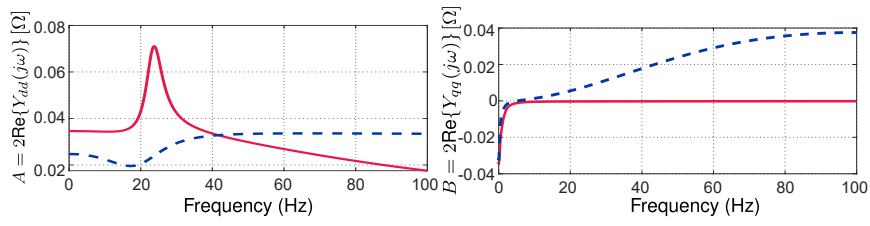

(b)

Fig. 12. Comparison between the passivity with (dashed) and without modifications (bold) of the vector controlled VSC for $A=2 \operatorname{Re}\left\{Y_{d d}(j \omega)\right\}$ and $B=2 \operatorname{Re}\left\{Y_{q q}(j \omega)\right\}$ (a) rectification (b) inversion

Fig. 12 shows a comparison between the passivity with and without modifications for the case of all-pass feedforward filters. As expected, slight improvements on the $d$-axis in rectifying operation and better improvement in the inverting operation on $q$-axis.

To further verify the improvements of the modified VCC structure over the existing structure, Fig. 13 shows the impedance magnitude response characterization of the VSC with the conventional VCC structure, across the entire operating range of the converter. Overall, the characterization indicates the relative ease of transition from one operating point to another. The conventional VCC structure shows several peaks and a non-smooth surface across the operating range from rectification $(1 \mathrm{pu})$ to inversion $(-1 \mathrm{pu})$. It also shows the dependence of VSC behavior on operating point and difficulty in transition for the conventional VCC structure under severe grid conditions. In the same manner, Fig. 14 shows the impedance response characterization of the modified structure. In general, the surface is remarkably smoother and void of peaks; this indicates an easier transition between operating points.

\section{Time-Domain Simulation And Analysis}

The previous sections relied on analytical derivations and frequency domain analysis. Nonlinear time-domain simulations are adopted to support previous analysis and validate the proposed modifications and exact capability limits. Several scenarios are simulated for both inverter and rectifier operations. Simulations are demonstrated first for an ac grid of SCR $=1$. In later cases, capability under any arbitrary grid strength 

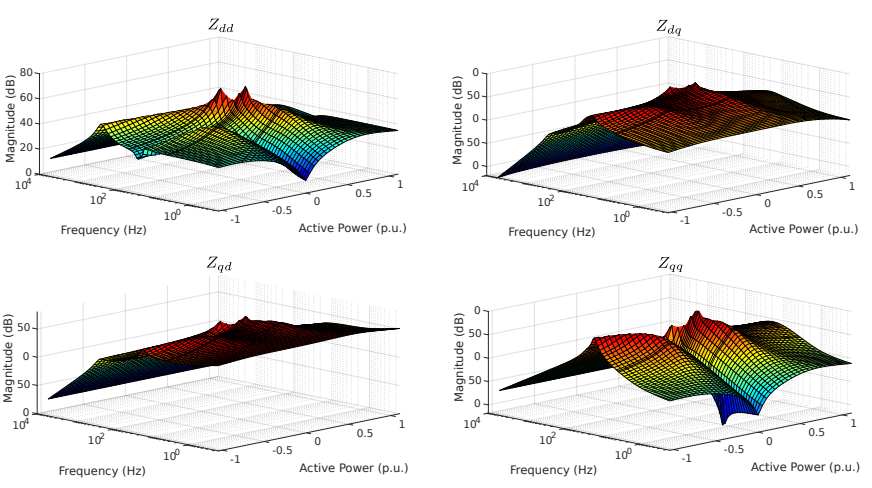

Fig. 13. VSC impedance magnitude characterization with the existing VCC across the entire operating range from $-1-1 \mathrm{pu}$
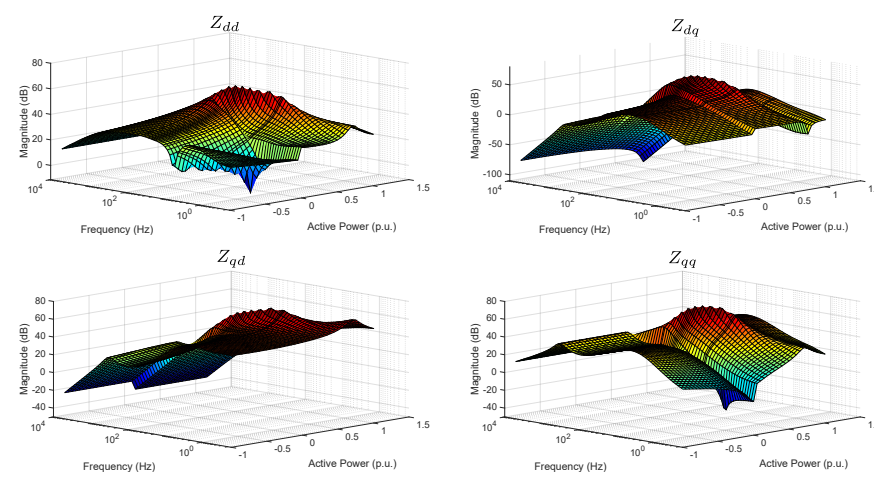

Fig. 14. VSC impedance magnitude characterization with the modified VCC across the entire operating range from $-1-1 \mathrm{pu}$

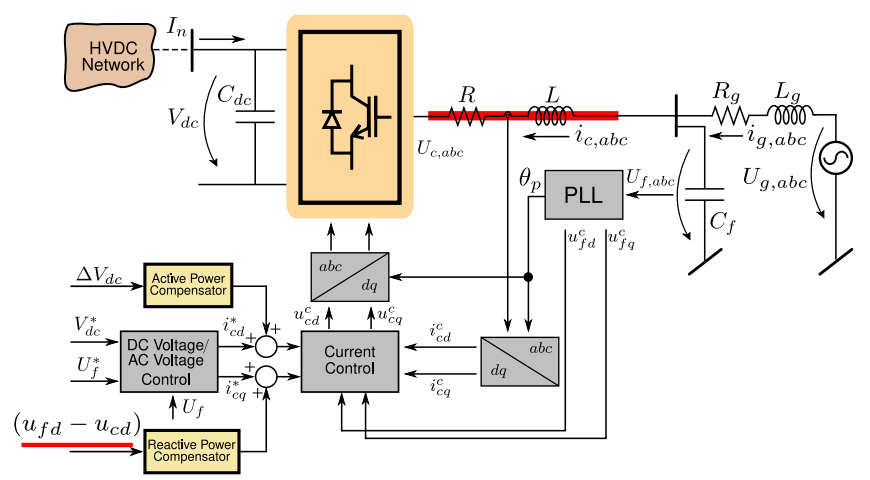

Fig. 15. Global overview of the overall system with the modified control structure

is demonstrated. Fig. 15 illustrates the global overview of the entire interconnected system and modified control structure as simulated in this section.

\section{A. Comparisons to Conventional Vector Control}

Figs. 16 and 17 shows the comparison of responses between the conventional vector control and the proposed modifications for the inversion and rectification stages respectively. The significant improvement over the existing structure particularly for inverter operation is clear considering the simplicity of modifications. With the conventional control, inverter operation is poorer than rectification due to the increased negative conductance in inversion as a result of the current sign.

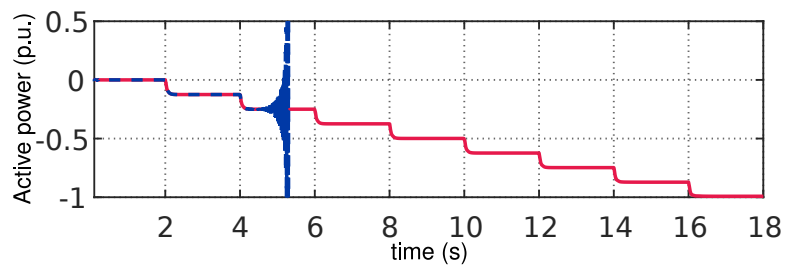

Fig. 16. Comparison between conventional vector control (dashed) and proposed modification (solid) in inverter operation

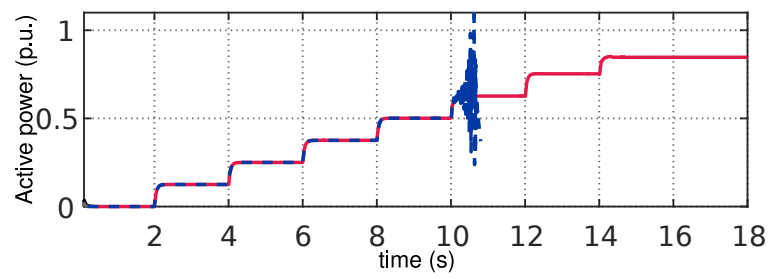

Fig. 17. Comparison between conventional vector control (dashed) and proposed modification (solid) in rectifier operation

Whereas in rectification there is an improvement over inversion due to the positive sign of current up to a region where negative conductance cannot be mitigated by the positive current sign. With the modified loops, inversion is possible up to $-1 \mathrm{pu}$ from $-0.3 \mathrm{pu}$ with the existing loop. In rectification, capability extends to $0.85 \mathrm{pu}$ from $0.6 \mathrm{pu}$ with the existing loop. This indicates an extended capability with the modified loop.

\section{B. Capability Under Varying Operating Conditions}

To demonstrate the capabilities of the modified vector current-controlled VSC, Fig. 18 shows the time-domain responses of active power, direct and alternating voltages over persistent step changes in dc link power across inverter operation. It shows the efficacy of variables in tracking the desired references up to -1 pu active power limit within a response time of $20 \mathrm{~ms}$. It is also seen that although the alternating and direct voltage responses consist of slight oscillations and magnitude deviation during the instant of step changes, deviations are well within $1 \%$ for direct voltage and $1.5 \%$ for alternating voltage, and oscillations are well-damped without significant degradation.

Likewise for the rectifier operation, Fig. 19 shows the similar responses. As can be clearly observed, with the proposed modification, rectification is only possible up to $\approx 0.85$ pu The inability to go further is the result of severe reactive power requirements under in rectifier operation compared to inverter operation. To further demonstrate the active power capabilities with the proposed modifications in rectifier operation under an improved grid strength, Fig. 20 shows the comparison of active power capabilities under SCR $=1$ and $\mathrm{SCR}=1.5$ showing that the rated power capability is possible with $\mathrm{SCR}=1.5$ with a limited reactive power support at the PCC. This is due to a considerable reduction in reactive requirements from the VSC under SCR $=1.5$ allowing more room for active power

and slight overrating of the converter to allow up to 1.02 pu of active power. 


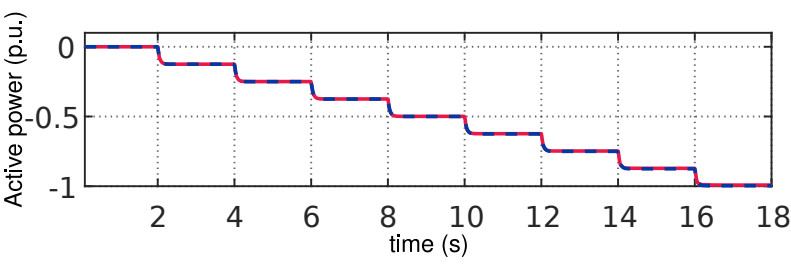

(a)

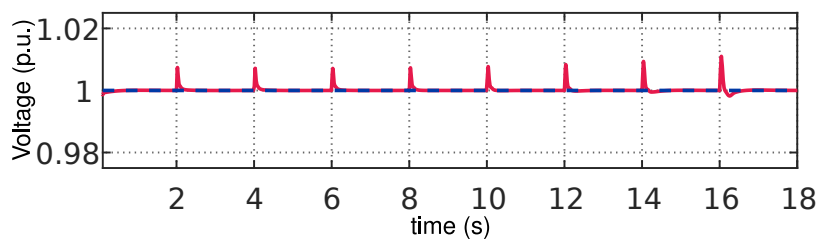

(b)

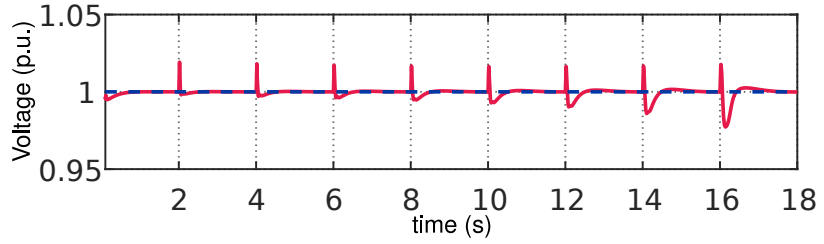

(c)

Fig. 18. Responses of controlled variables under varying operating points in inverter operation with the proposed modifications. Reference (dashed), measurement (solid) (a) active power (b) direct voltage (c) alternating voltage

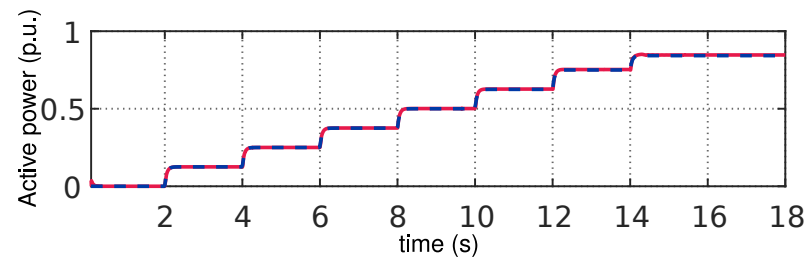

(a)

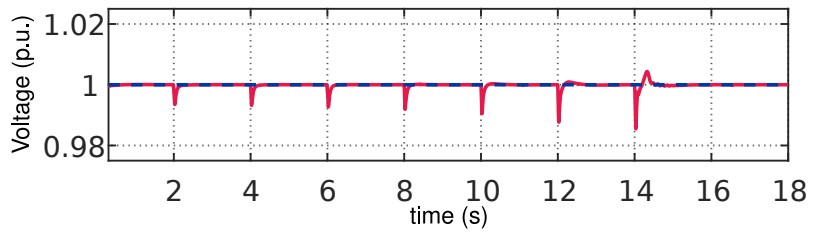

(b)

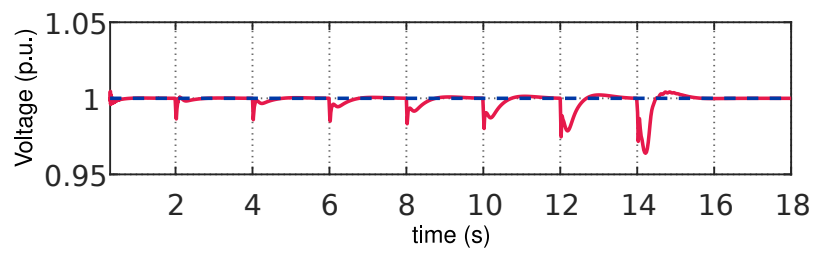

(c)

Fig. 19. Responses of controlled variables under varying operating points in rectifier operation with the proposed modifications. Reference (dashed), measurement (solid) (a) active power (b) direct voltage (c) alternating voltage

\section{Reactive Behavior Under Changing Grid Conditions}

Fig. 21 demonstrates the converter reactive behavior with proposed modifications under changing grid strength in in-

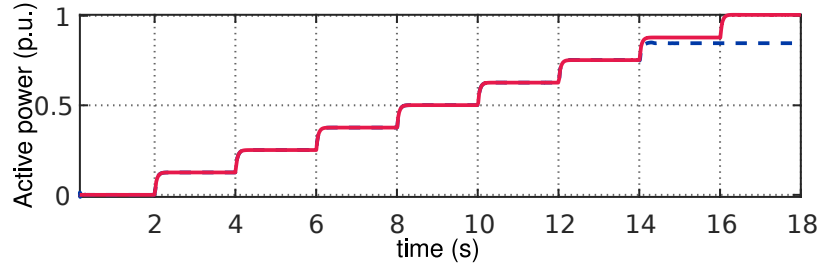

Fig. 20. Comparison of active power capabilities under SCR 1 (dashed) and 1.5 (solid) with limited reactive power support at the PCC

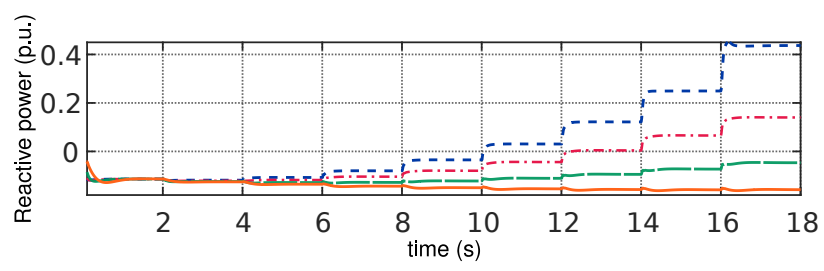

(a)

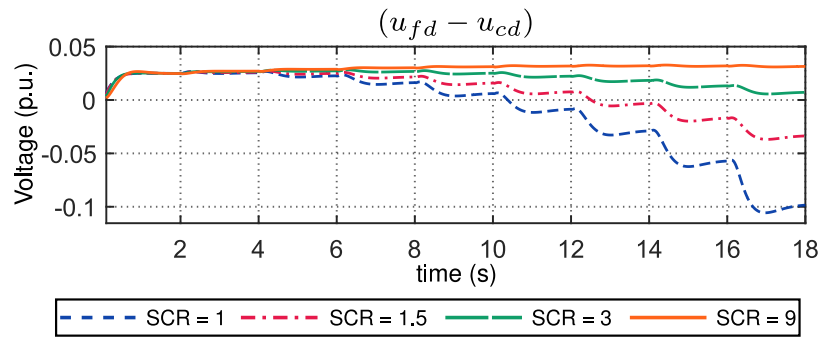

(b)

Fig. 21. Reactive behavior under varying grid condition in inversion (a) Reactive power (b) voltage difference $\left(u_{f d}-u_{c d}\right)$ across filter on $d$-axis

verter operation. It shows clearly in (a) the reactive requirements demanded from the converter under SCR = 1 compared to $\mathrm{SCR}=1.5$ given both grids are still relatively very weak. As grid conditions improve towards a stronger grid, reactive support required of converter drops considerably. Additionally, (b) supports the analysis in the previous section on the sign of $\left(u_{f d}-u_{c d}\right)$ in weak grids. As can be observed, for a fairly strong to strong grid ( $\mathrm{SCR}>3$ ), the difference is positive across all operating points and reactive requirements are easily managed. However, the difference becomes negative at (SCR $<3$ ) as the grid is severely weakened and the magnitude of negativity increases with increasing active power. The same behavior applies to rectifier operation.

\section{CONCLUSIONS}

A detailed MIMO impedance model of a vector controlled VSC in alternating and direct voltage control mode has been derived. The concept of passivity/dissipativity has been applied to obtain insights into variables that may contribute to detrimental behavior. Interestingly, several variables were found in which under fairly strong grids (SCR $\geq 3$ ) behave as expected. However, under weak grids, the same variables significantly contribute to instability. Hence, a solution is to feedforward these variables or combinations thereof through a modification of the existing structure, thereby partially eliminating their influence. From a dissipativity point of view, this significantly reduces the deficit in internal energy balance. In summary, it 
is found that the simple modification proposed significantly improves the active power capability from $-1 \mathrm{pu}$ in inversion to $0.85 \mathrm{pu}$ in rectification in very weak grid condition (SCR $=1$ ), and across the entire operating range once SCR improves to as low as 1.5, without changing the basic structure of the vector control. Results indicate the performance under any arbitrary grid strength and demonstrate that complex strategies are not required.

\section{REFERENCES}

[1] M. Barnes and A. Beddard, "Voltage source converter HVDC links the state of the art and issues going forward," Energy Procedia, vol. 24, pp. 108 - 122, 2012, selected papers from Deep Sea Offshore Wind R\& D Conference, Trondheim, Norway, 19-20 Jan. 2012.

[2] N. Ahmed, A. Haider, D. V. Hertem, L. Zhang, and H. Nee, "Prospects and challenges of future HVDC supergrids with modular multilevel converters," in Proceedings of the 2011 14th European Conference on Power Electronics and Applications, Aug. 2011, pp. 1-10.

[3] J. Rocabert, A. Luna, F. Blaabjerg, and P. Rodrguez, "Control of power converters in ac microgrids," IEEE Transactions on Power Electronics, vol. 27, no. 11, pp. 4734-4749, Nov. 2012.

[4] L. Zhang, L. Harnefors, and H. Nee, "Power-synchronization control of grid-connected voltage-source converters," IEEE Transactions on Power Systems, vol. 25, no. 2, pp. 809-820, May 2010.

[5] L. Zhang, "Modeling and control of VSC-HVDC links connected to weak ac systems," Ph.D. dissertation, KTH, 2010.

[6] J. Z. Zhou, H. Ding, S. Fan, Y. Zhang, and A. M. Gole, "Impact of short-circuit ratio and phase-locked-loop parameters on the small-signal behavior of a VSC-HVDC converter," IEEE Transactions on Power Delivery, vol. 29, no. 5, pp. 2287-2296, Oct. 2014.

[7] Y. Huang, X. Yuan, J. Hu, and P. Zhou, "Modeling of VSC connected to weak grid for stability analysis of DC-link voltage control," IEEE Journal of Emerging and Selected Topics in Power Electronics, vol. 3, no. 4, pp. 1193-1204, Dec. 2015.

[8] D. Dong, B. Wen, D. Boroyevich, P. Mattavelli, and Y. Xue, "Analysis of phase-locked loop low-frequency stability in three-phase grid-connected power converters considering impedance interactions," IEEE Transactions on Industrial Electronics, vol. 62, no. 1, pp. 310-321, Jan. 2015.

[9] S.-K. Chung, "A phase tracking system for three phase utility interface inverters," IEEE Transactions on Power Electronics, vol. 15, no. 3, pp. 431-438, May 2000.

[10] P. Rodriguez, J. Pou, J. Bergas, J. I. Candela, R. P. Burgos, and D. Boroyevich, "Decoupled double synchronous reference frame pll for power converters control," IEEE Transactions on Power Electronics, vol. 22, no. 2, pp. 584-592, Mar. 2007.

[11] K. Lee, J. Lee, D. Shin, D. Yoo, and H. Kim, "A novel grid synchronization pll method based on adaptive low-pass notch filter for gridconnected pcs," IEEE Transactions on Industrial Electronics, vol. 61, no. 1, pp. 292-301, Jan. 2014.

[12] R. Teodorescu, M. Liserre, and P. Rodriguez, Grid converters for photovoltaic and wind power systems. John Wiley \& Sons, 2011, vol. 29.

[13] M. F. M. Arani and Y. A. I. Mohamed, "Analysis and performance enhancement of vector-controlled VSC in HVDC links connected to very weak grids," IEEE Transactions on Power Systems, vol. 32, no. 1, pp. 684-693, Jan. 2017.

[14] S. Sang, N. Gao, X. Cai, and R. Li, "A novel power-voltage control strategy for the grid-tied inverter to raise the rated power injection level in a weak grid," IEEE Journal of Emerging and Selected Topics in Power Electronics, vol. 6, no. 1, pp. 219-232, March 2018.

[15] R. Aouini, B. Marinescu, K. B. Kilani, and M. Elleuch, "Stability improvement of the interconnection of weak AC zones by synchronverterbased HVDC link," Electric Power Systems Research, vol. 142, pp. 112 $-124,2017$.

[16] L. Zhang, L. Harnefors, and H. Nee, "Interconnection of two very weak AC systems by VSC-HVDC links using power-synchronization control," IEEE Transactions on Power Systems, vol. 26, no. 1, pp. 344-355, Feb 2011.

[17] Y. Gui, X. Wang, H. Wu, and F. Blaabjerg, "Voltage-modulated direct power control for a weak grid-connected voltage source inverters," IEEE Transactions on Power Electronics, vol. 34, no. 11, pp. 11383-11395, Nov 2019.
[18] L. Harnefors, M. Bongiorno, and S. Lundberg, "Input-admittance calculation and shaping for controlled voltage-source converters," IEEE Transactions on Industrial Electronics, vol. 54, no. 6, pp. 3323-3334, Dec. 2007.

[19] N. P. W. Strachan and D. Jovcic, "Stability of a variable-speed permanent magnet wind generator with weak AC grids," IEEE Transactions on Power Delivery, vol. 25, no. 4, pp. 2779-2788, Oct. 2010.

[20] A. Egea-Alvarez, S. Fekriasl, F. Hassan, and O. Gomis-Bellmunt, "Advanced vector control for voltage source converters connected to weak grids," IEEE Transactions on Power Systems, vol. 30, no. 6, pp. 3072-3081, Nov. 2015.

[21] Y. Huang and D. Wang, "Effect of control-loops interactions on power stability limits of VSC integrated to AC system," IEEE Transactions on Power Delivery, vol. 33, no. 1, pp. 301-310, Feb 2018.

[22] G. Wu, J. Liang, X. Zhou, Y. Li, A. Egea-Alvarez, G. Li, H. Peng, and X. Zhang, "Analysis and design of vector control for VSC-HVDC connected to weak grids," CSEE Journal of Power and Energy Systems, vol. 3, no. 2, pp. 115-124, Jun. 2017.

[23] X. Zhang, D. Xia, Z. Fu, G. Wang, and D. Xu, "An improved feedforward control method considering PLL dynamics to improve weak grid stability of grid-connected inverters," IEEE Transactions on Industry Applications, vol. 54, no. 5, pp. 5143-5151, Sep. 2018.

[24] S. Lu, Z. Xu, L. Xiao, W. Jiang, and X. Bie, "Evaluation and enhancement of control strategies for VSC stations under weak grid strengths,' IEEE Transactions on Power Systems, vol. 33, no. 2, pp. 1836-1847, March 2018.

[25] M. Davari and Y. A. I. Mohamed, "Robust vector control of a very weakgrid-connected voltage-source converter considering the phase-locked loop dynamics," IEEE Transactions on Power Electronics, vol. 32, no. 2, pp. 977-994, Feb 2017.

[26] O. Brune, "Synthesis of a finite two-terminal network whose drivingpoint impedance is a prescribed function of frequency," Journal of Mathematics and Physics, vol. 10, no. 1-4, pp. 191-236, 1931.

[27] R. Ortega, J. A. L. Perez, P. J. Nicklasson, and H. J. Sira-Ramirez, Passivity-based control of Euler-Lagrange systems: mechanical, electrical and electromechanical applications. Springer Science \& Business Media, 2013

[28] J. C. Willems, "Dissipative dynamical systems," European Journal of Control, vol. 13, no. 2, pp. $134-151,2007$

[29] L. Harnefors, L. Zhang, and M. Bongiorno, "Frequency-domain passivity-based current controller design," IET Power Electronics, vol. 1, no. 4 , pp. 455-465, Dec. 2008

[30] L. Harnefors, A. G. Yepes, A. Vidal, and J. Doval-Gandoy, "Passivitybased controller design of grid-connected vscs for prevention of electrical resonance instability," IEEE Transactions on Industrial Electronics, vol. 62, no. 2, pp. 702-710, Feb. 2015.

[31] X. Wang, F. Blaabjerg, and P. C. Loh, "Passivity-based stability analysis and damping injection for multiparalleled VSCs with LCL filters," IEEE Transactions on Power Electronics, vol. 32, no. 11, pp. 8922-8935, Nov 2017.

[32] R. Ortega, A. van der Schaft, B. Maschke, and G. Escobar, "Interconnection and damping assignment passivity-based control of port-controlled hamiltonian systems," Automatica, vol. 38, no. 4, pp. 585 - 596, 2002.

[33] L. Harnefors, X. Wang, A. G. Yepes, and F. Blaabjerg, "Passivitybased stability assessment of grid-connected VSCs-an overview," IEEE Journal of Emerging and Selected Topics in Power Electronics, vol. 4, no. 1, pp. 116-125, Mar. 2016.

[34] A. J. Agbemuko, J. L. Domínguez-García, E. Prieto-Araujo, and $\mathrm{O}$. Gomis-Bellmunt, "Dynamic modelling and interaction analysis of multi-terminal VSC-HVDC grids through an impedance-based approach," International Journal of Electrical Power \& Energy Systems, vol. 113, pp. $874-887,2019$.

[35] M. Xia, P. J. Antsaklis, V. Gupta, and F. Zhu, "Passivity and dissipativity analysis of a system and its approximation," IEEE Transactions on Automatic Control, vol. 62, no. 2, pp. 620-635, Feb 2017.

[36] L. Harnefors, "Modeling of three-phase dynamic systems using complex transfer functions and transfer matrices," IEEE Transactions on Industrial Electronics, vol. 54, no. 4, pp. 2239-2248, Aug. 2007.

[37] G. Strang, Linear Algebra and Its Applications. Thomson, Brooks/Cole, 2006.

[38] D. Wang, L. Liang, L. Shi, J. Hu, and Y. Hou, "Analysis of modal resonance between PLL and DC-link voltage control in weak-grid tied VSCs," IEEE Transactions on Power Systems, vol. 34, no. 2, pp. 11271138, March 2019. 


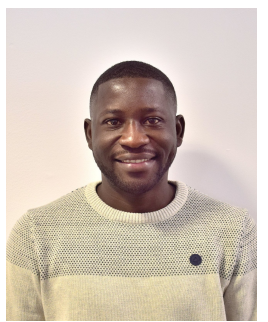

Adedotun J. Agbemuko (S'16-M'20) received the BEng. degree in electrical engineering from the Federal University of Technology, Minna (FUT Minna), Nigeria, in 2012, the MSc. degree in electrical engineering from the Delft University of Technology, Delft (TU Delft), the Netherlands, in 2016, and the Ph.D. degree (cum laude) in electrical engineering from Technical University of Catalonia (UPC), Barcelona, Spain in 2019. Since 2016, he has been with the power systems research group at Institut de Recerca en Energia de Catalunya (IREC). His research interests include modelling, network-scale interaction analysis and control of grid-connected converters, statistical-physics based modelling, and control of large-scale hybrid AC/DC grids including multi-terminal HVDC grids.

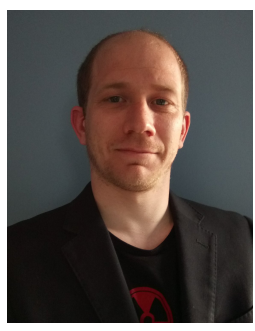

Jose Luis Domínguez-García received the B.Sc and M.Sc. degrees in industrial engineering from the School of Industrial Engineering of Barcelona, Universitat Politécnica de Catalunya (UPC), Barcelona, Spain, in 2009, the masters thesis in Oulun Yliopisto, Oulu, Finland, and the Ph.D. degree (cum laude) in electrical engineering from UPC, in 2013. He was a Researcher Visitor with the Institute of Energy, Cardiff University, and the University of Strathclyde, U.K., in 2011 and 2016, respectively. In 2010, he joined IREC, Barcelona, Spain, as a Researcher, and is currently the Head of Power Systems Group. He has been awarded the Distinguished Visiting Fellowship from the Royal Academy of Engineering. He has authored more than 25 journal papers and coauthored 1 patent. He actively participates and coordinates several industrial and research projects focused on wind power integration, power system stability, and offshore grids design and control. His research interests include grid integration, control and modelling of renewable energies, transmission and distribution grid management, power converters, and power system dynamics. He was the recipient of the Outstanding Ph.D. Thesis award from UPC in 2015.

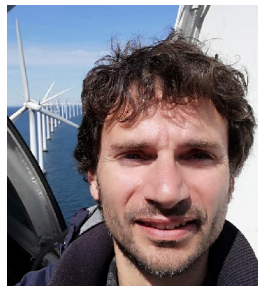

Oriol Gomis-Bellmunt (S'05-M'07-SM'12) received the degree in industrial engineering from the School of Industrial Engineering of Barcelona (ETSEIB), Technical University of Catalonia (UPC), Barcelona, Spain, in 2001 and the Ph.D. degree in electrical engineering from the UPC in 2007. In 1999, he joined Engitrol S.L. where he worked as Project Engineer in the automation and control industry. Since 2004, he has been with the Electrical Engineering Department, UPC where he is a Professor and participates in the CITCEA-UPC Research Group. Since 2020, he is an ICREA Academia researcher. His research interests include the fields linked with electrical machines, power electronics, and renewable energy integration in power systems.

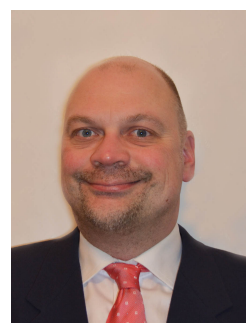

Lennart Harnefors (S'93-M'97-SM'07-F'17) received the M.Sc., Licentiate, and Ph.D. degrees in electrical engineering from the Royal Institute of Technology (KTH), Stockholm, Sweden, and the Docent (D.Sc.) degree in industrial automation from Lund University, Lund, Sweden, in 1993, 1995, 1997, and 2000, respectively. Between 1994-2005, he was with Mälardalen University, Västerås, Sweden, from 2001 as a Professor of electrical engineering. Between 2001-2005, he was, in addition, a part-time Visiting Professor of electrical drives with Chalmers University of Technology, Göteborg, Sweden. In 2005, he joined ABB, HVDC Product Group, Ludvika, Sweden, where he led the control development for cascaded-two-level-converter HVDC Light. In 2012, he joined ABB, Corporate Research, Västerås, Sweden, where he was appointed as a Senior Principal Scientist in 2013. He is, in addition, a part-time Adjunct Professor of power electronics with KTH since 2011.

Dr. Harnefors is an Associate Editor of IET Electric Power Applications. He was certified as a 2018 Outstanding Reviewer of the IEEE TRANSACTIONS ON POWER ELECTRONICS. His research interests include control and dynamic analysis of power electronic systems, particularly grid-connected converters and ac drives. 